\title{
Trapping and guiding microparticles with morphing autofocusing Airy beams
}

\author{
Peng Zhang, ${ }^{1}$ Jai Prakash, ${ }^{1}$ Ze Zhang, ${ }^{1,2,3}$ Matthew S. Mills, ${ }^{2}$ Nikolaos K. Efremidis, ${ }^{4}$ \\ Demetrios N. Christodoulides, ${ }^{2}$ and Zhigang Chen ${ }^{1,5, *}$ \\ ${ }^{1}$ Department of Physics and Astronomy, San Francisco State University, San Francisco, California 94132 \\ ${ }^{2}$ CREOL/College of Optics, University of Central Florida, Orlando, Florida 32816 \\ ${ }^{3}$ National Key Laboratory of Tunable Laser Technology, Harbin Institute of Technology, Harbin 150080, China \\ ${ }^{4}$ Department of Applied Mathematics, University of Crete, 71409 Heraklion, Crete, Greece \\ ${ }^{5}$ TEDA Applied Physics School, Nankai University, Tianjin 300457, China \\ *Corresponding author: zhigang@sfsu.edu
}

Received May 31, 2011; revised June 23, 2011; accepted June 23, 2011; posted June 27, 2011 (Doc. ID 148366); published July 25, 2011

We observe optical trapping and manipulation of dielectric microparticles using autofocusing radially symmetric Airy beams. This is accomplished by exploiting either the inward or outward transverse acceleration associated with their chirped wavefronts. We experimentally demonstrate, for the first time to our knowledge, that such Airy beams morph into nondiffracting Bessel beams in their far-field. Furthermore, the ability of guiding and transporting microparticles along the primary rings of this class of beams is explored. (c) 2011 Optical Society of America OCIS codes: $350.4855,350.5500,260.6042,050.1940$.

Beam focusing has always been a subject of practical importance. For many applications it is desirable to have a wavefront's energy abruptly autofocused right onto a target while maintaining a low intensity profile up until that point. This feature could be useful, for example, in biomedical treatments and other nonlinear settings such as optical filamentation. Lately, autofocusing waves based on Airy beams [1,2] has been proposed [3,4] and observed $[\underline{5}, 6]$. These field configurations rely on radially symmetric or circular Airy beams (CABs) and they can be established entirely on linear effects, i.e., without invoking any nonlinear self-focusing processes [3]. In addition, a recent theoretical study has also indicated that a superposition of Airy wavelets can asymptotically "morph" into Bessel beams in their far-field [7]. In this Letter, we report on our observation of autofocusing phenomenon for radially symmetric Airy beams with either inward or outward accelerations, and experimentally confirm for the first time this morphing behavior and show that they indeed become Bessel beams in their respective far-fields. Moreover, we also examine the trapping and guiding of microparticles using such CABs.

A circular Airy beam can exhibit either an inward or outward acceleration and the direction of acceleration is always determined by the radial Airy tails. If the tails point outwards, the $\mathrm{CAB}$ primary ring accelerates inwards and vice versa. First, let us generate an inward accelerating CAB by use of an off-axis hologram. Such a hologram is manifested by computing the interference between an autofocusing Airy wave and a plane wave. Similar methods with Moiré techniques have been used in the past to generate optical rotating beams and optical bottle beams [,ㅛ, 9 ]. The experimental setup illustrated in Fig. 1 is similar to that used in our prior demonstrations of linear and nonlinear control of two-dimensional (2D) Airy beams $[10,11]$. As in the case for 2D Airy beams, the $\mathrm{CAB}$ is generated with the assistance of a spatial light modulator (SLM). Specifically, a Gaussian beam from a Coherent Verdi laser $(\lambda=532 \mathrm{~nm})$ is sent through the SLM programmed with the desired hologram and then a $4 \mathrm{f}$ system with a spatial filter turns the Gaussian beam into an inward accelerating CAB. Theoretical results corresponding to this arrangement are shown in Fig. 2.

It is clear that the beam abruptly autofocuses after a certain distance of propagation. This "focal length" directly depends on the diameter and acceleration of the initial profile. Although the CAB has a number of outer rings, the brightest ring contains approximately half of the total energy.

Note that the bright rings of the inward accelerating $\mathrm{CAB}$ move on a parabolic trajectory [as shown between planes a-c in Fig. 2(e)] until autofocusing occurs. After this point, our simulations indicate that the beam profile asymptotically takes the form of a Bessel beam. In other words, the $\mathrm{CAB}$ morphs into a first order nondiffracting Bessel field.

Experimental data corresponding to the theoretical results of Fig. 2 are displayed in Fig. 3 . As before, the bottom panel shows the autofocusing beam from a side propagating view and the top panels display snapshots of transverse patterns at different propagation distances. For better visualization, the intensities in Figs. 3(a)-3(d) have all been scaled to the same peak intensity; however, the intensity pattern without normalization would illustrate the drastic increase of peak intensity near the "focal

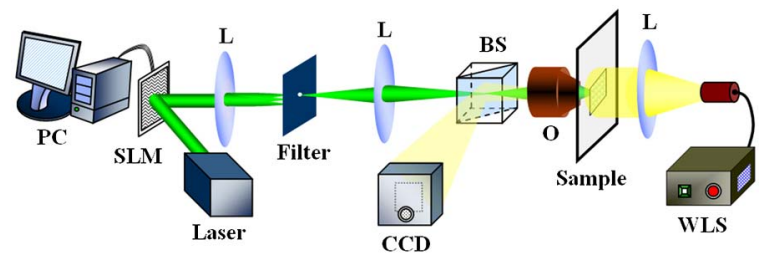

Fig. 1. (Color online) Schematic of experimental setup for generation of circular Airy beams and their use in particle trapping. SLM: spatial light modulator; $L$ : lens; BS: beam splitter; $O$ : objective lens; WLS: white-light source. Spatial filter and $4 f$ system is used for generation desired CABs from computergenerated holograms. 

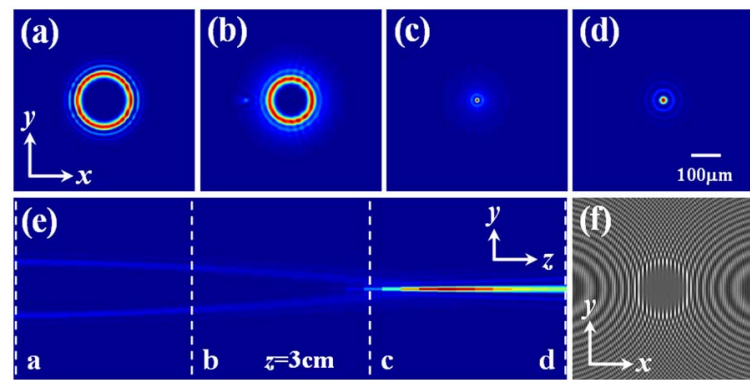

Fig. 2. (Color online) Generation of autofocusing beam with computer-generated holograms. (a)-(d): Snapshots of the transverse intensity patterns taken at planes as marked by dashed white lines in (e), the side-view of the autofocusing beam propagating along $z$; (f) typical off-axis hologram.

point" of the CAB. In Fig. 3(e), the propagation length is about $3 \mathrm{~cm}$ and the beam size changed from $600 \mu \mathrm{m}$ to $20 \mu \mathrm{m}$. Our findings show a good agreement between theoretical and experimental results.

Using a similar approach, we also created radially symmetric Airy beams with outward acceleration. In this case, the $\mathrm{CAB}$ has its brightest intensity located in the outermost ring with decreasing intensity in the radial direction towards the beam center. Corresponding numerical and experimental outcomes are juxtaposed in Fig. 4 . Intuitively, the $\mathrm{CAB}$ generated this way would be expected to accelerate sideways in all directions, as seen from the decaying intensity in side-view of beam propagation displayed in Fig. 4(e). Thus, the beam actually bends outward and experiences autodefocusing rather than focusing during propagation. Yet, in spite of this "defocusing" behavior, the field again asymptotically forms a Bessel beam.

To better understand this odd behavior, e.g. why in both cases a CAB asymptotically morphs into a Bessel beam, we consider the far-field Fraunhofer diffraction pattern, which is given by the Hankel transform of the initial field distribution. In this case, the optical field envelope is described by:

$$
E(\rho, z)=-\frac{i k}{z} \exp \left(\frac{i k \rho^{2}}{2 z}\right) \int_{0}^{\infty} r E(r, 0) J_{0}\left(\frac{k \rho r}{z}\right) \mathrm{d} r
$$

where $E(r, 0)$ represents the initial radial Airy profile, i.e.,

$$
E(r, 0)=A i\left[ \pm\left(\frac{r_{0}-r}{w}\right)\right] \exp \left[ \pm \alpha\left(\frac{r_{0}-r}{w}\right)\right] .
$$
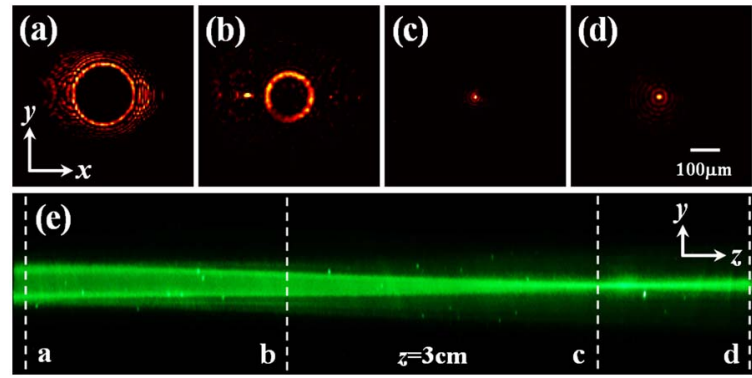

Fig. 3. (Color online) (a)-(d) Experimental snapshots of transverse intensity patterns of the autofocusing beam (contrast enhanced) taken at different planes as marked in (e), the direct side-view photography of the beam taken from scattered light.
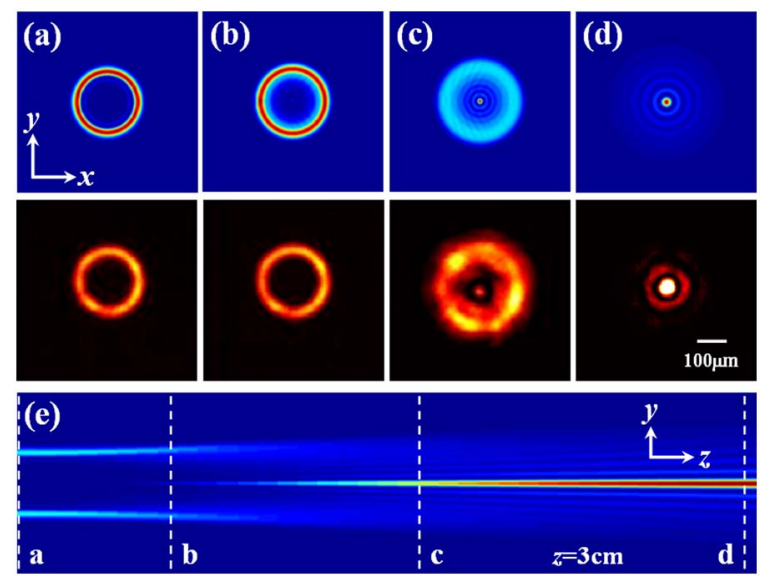

Fig. 4. (Color online) Generation of circular Airy beam with initial outward acceleration. (a)-(d): Intensity snapshots from simulation (first row) and experiment (second row) taken at planes as marked in (e) the side-view propagation along $z$. Notice that, overall, the beam bends outward rather than inward as seen from the "tails" and the transition into Bessel beam is less abrupt as compared with that in Fig. 2 .

In Eq. (2), the \pm arrangement corresponds to inward and outward acceleration of CABs, respectively, and $w$ is associated with the width of the main Airy lobe. In both cases, the Airy structure is exponentially truncated $[1,2]$ at a rate $\alpha$ and located around $r_{0}$, the radial position of the main Airy ring. Strictly speaking, the integral in Eq. (1) cannot be exactly evaluated. Yet, we can develop a fairly accurate description of this process provided that the main radial lobe of this $\mathrm{CAB}$ is approximated by a delta-ring of amplitude $A_{0}$, e.g., $E(r, 0) \approx A_{0} \delta\left(r-r_{0}\right)$. In this case, the integral of Eq. (1) can be readily evaluated and is given by:

$$
E(\rho, z)=-\frac{i k}{z} \exp \left(\frac{i k \rho^{2}}{2 z}\right) r_{0} A_{0} J_{0}\left(\frac{k \rho r_{0}}{z}\right) .
$$

By considering the Eqs. (1)-(3) at $\rho=0$, we can then determine the amplitude $A_{0}$ :

$$
A_{0} \approx w \exp \left(\frac{\alpha^{3}}{3}\right)\left(1-\frac{w \alpha^{2}}{r_{0}}\right)
$$

Figure 5 displays our theoretical results of this far-field Bessel-morphing behavior at a normalized diffraction distance of $z /\left(k w^{2}\right)=10$.

One of the proposed applications of accelerating Airy beams is for optical manipulation of particles $[12,13]$. Below, we explore such a possibility using our circular

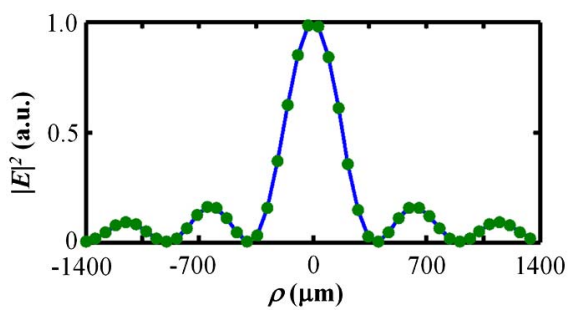

Fig. 5. (Color online) Output given by Eq. (3) (solid circles) and from an exact numerical calculation of Eq. (1) (solid line) for parameters $\alpha=0.05, w=70 \mu \mathrm{m}, r_{0}=300 \mu \mathrm{m}$. 


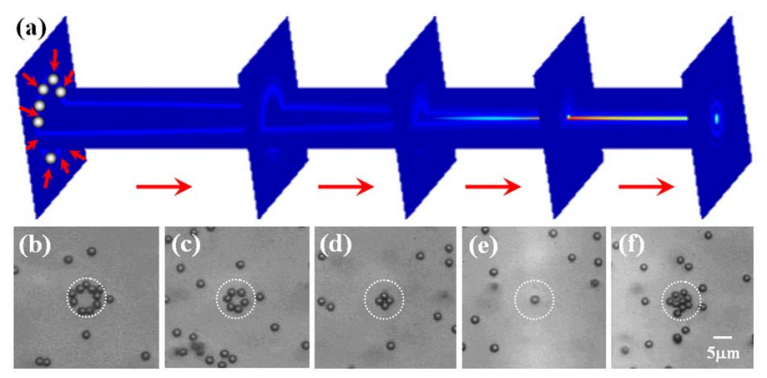

Fig. 6. (Color online) Experimental demonstration of particle guidance with a circular autofocusing beam. (a) Illustration of particle motion through a tapered particle guide; (b)-(f) Snapshots of trapped microparticles from videos taken when the particles are trapped at different transverse planes (see Media 1 for an example). Dashed circles serve as a reference while the arrows illustrate the movement of the particles.

autofocusing Airy beams. The CAB as generated in Fig. 3 is sent into an optical tweezers-like setting as shown in Fig. 1. Because of the autofocusing nature of the beam, the objective lens used here $(40 X, \mathrm{NA}=0.65)$ has a much weaker amplification as compared to those used in normal settings of optical tweezers. The sample consists of $2 \mu \mathrm{m}$ polystyrene beads in water solution sandwiched between two thin microslide glass plates. Imaging of the particles is achieved by illuminating the sample with a white-light beam from the opposite direction as captured by a CCD camera. Typical experimental results are shown in Fig. $\underline{6}$, where the top panel illustrates how the particles are trapped and transported along the autofocusing beam, while the bottom panels are snapshots of the microparticles trapped at different longitudinal positions. To visualize the particle trapping at a different transverse plane, we gradually move our sample along the longitudinal direction, so the particles are always pushed against the rear glass plate, as monitored by the CCD camera. This serves as an indirect observation of particle movement along the longitudinal direction since side-view imaging is difficult in our experiment. As seen from the media file, the particles tend to form ring patterns at different transverse planes to match the primary ring of the $\mathrm{CAB}$. When the radius of the ring decreases, less particles can stay trapped in the ring. At the "focal point", when the beam abruptly focuses into a bright spot, only one particle can be trapped stably [Fig. 6(e)] by the beam while other particles can move around the beam. After the "focal point", the focused Airy beam turns into a Bessel beam, in which more particles can be attracted to the high-intensity region of the central bright spot as well as adjacent rings. This suggests that the $\mathrm{CAB}$ can be used as a tapered channel guide for microparticles.

In summary, we have shown that circular autofocusing Airy beams can be generated and morphed into Bessel beams. By use of the autofocusing beams with inward acceleration, we have demonstrated optical guiding of nonabsorbing microparticles. We mention that such optical manipulation relies on gradient forces as in optical tweezers, but different from recent demonstrations of optical trapping of airborne particles based on photophoretic forces $[9,14,15]$. Our results with optical autofocusing beams bring about new possibilities for trapping and guiding microparticles in a controllable fashion, which may find applications in optical, biological, and atmospheric sciences.

This work was supported by the National Science Foundation (NSF), the Air Force Office of Scientific Research (USAFOSR) (FA9550-09-1-0474), Multi-discipline research initiative (MURI) grant (FA9550-10-1-0561), an internal award at San Francisco State University, and by the 973 Program in China.

\section{References}

1. G. A. Siviloglou and D. N. Christodoulides, Opt. Lett. 32, 979 (2007).

2. G. A. Siviloglou, J. Broky, A. Dogariu, and D. N. Christodoulides, Phys. Rev. Lett. 99, 213901 (2007).

3. N. K. Efremidis and D. N. Christodoulides, Opt. Lett. 35, 4045 (2010).

4. I. Chremmos, N. K. Efremidis, and D. N. Christodoulides, Opt. Lett. 36, 1890 (2011).

5. P. Zhang, J. Prakash, Z. Zhang, Y. Hu, N. K. Efremidis, V. Kajorndejnukul, D. N. Christodoulides, and Z. Chen, "Observation of auto-focusing radially symmetric Airy beams," presented at Conference on Lasers and Electro-Optics: Quantum Electronics and Laser Science, paper QThS7 (May 1-May 6, 2011).

6. D. G. Papazoglou, N. K. Efremidis, D. N. Christodoulides, and S. Tzortzakis, Opt. Lett. 36, 1842 (2011).

7. C. Hwang, K. Kim, and B. Lee, Opt. Express 19, 7356 (2011).

8. P. Zhang, S. Huang, Y. Hu, D. Hernandez, and Z. Chen, Opt. Lett. 35, 3129 (2010).

9. P. Zhang, Z. Zhang, J. Prakash, S. Huang, D. Hernandez, M. Salazar, D. N. Christodoulides, and Z. Chen, Opt. Lett. 36, 1491 (2011).

10. Y. Hu, P. Zhang, C. Lou, S. Huang, J. Xu, and Z. Chen, Opt. Lett. 35, 2260 (2010).

11. Y. Hu, S. Huang, P. Zhang, C. Lou, J. Xu, and Z. Chen, Opt. Lett. 35, 3952 (2010).

12. J. Baumgartl, M. Mazilu, and K. Dholakia, Nat. Photonics 2, 675 (2008).

13. H. Cheng, W. Zang, W. Zhou, and J. Tian, Opt. Express 18, 20384 (2010).

14. V. G. Shvedov, A. S. Desyatnikov, A. V. Rode, W. Krolikowski, and Y. S. Kivshar, Opt. Express 17, 5743 (2009).

15. V. G. Shvedov, A. V. Rode, Y. V. Izdebskaya, A. S. Desyatnikov, W. Krolikowski, and Y. S. Kivshar, Phys. Rev. Lett. 105, 118103 (2010). 